\title{
Advances for 3D printing: Remote control system and multi-material solutions
}

\author{
Adrián Luque Luque \\ University of Jaén, \\ Spain \\ alluque@ujaen.es
}

\author{
Juan Manuel Jurado \\ Rodríguez \\ University of Jaén, \\ Spain \\ jjurado@ujaen.es
}

\author{
José Luis Cárdenas \\ Donoso \\ University of Jaén, \\ Spain \\ jcdonoso@ujaen.es
}

\author{
Francisco R. Feito \\ Higueruela \\ University of Jaén, \\ Spain \\ ffeito@ujaen.es
}

\begin{abstract}
Three-dimensional printing is an emerging manufacturing technology for many applications such as rapid prototyping, biomedical engineering and industrial designs. This paper aims to provide the implantation of a low-cost and extensible system in order to real-time monitor and modify 3D printing parameters. It is formed by a webbased platform and hardware components to manage and capture the printing process. In addition, we raise an overview about material properties in order to generate multi-material prototypes of bone tissue.
\end{abstract}

\section{Keywords}

3D printing, remote control system, multi-material, medical prototypes

\section{INTRODUCTION}

Additive manufacturing, commonly referred to as 3D printing has matured technically and is becoming more widespread for multiple application areas. Nowadays, the increasingly low cost and accessibility of threedimensional (3D) printers has caused the exploitation of this technology from multiple enterprises. 3D printing is capable of creating physical models with a high quality in a short time. In this context, a lot of printing materials have raised which satisfy different physical properties depending on its subsequent application. In this paper, we are focused on Fused Deposition Modeling (FDM) technique [1]. FDM is the most widely used due to there are many low-cost 3D printers which support this technology and its printing parameters are easy to set. In this case, 3D printed objects are built by selectively deposition material in a pre-defined path layerby-layer. However, FDM fabrication process has some limitations that must be known before any 3D printing. This technology belongs to the material extrusion family and can only be used with thermoplastic polymers through a filament form. Choosing the right type of material to print requires a high knowledge about its features and then, testing its behavior during the 3D printing. For this reason, we have used Octoprint application which provides a web interface for remote control of the $3 \mathrm{D}$ printer. It has been installed into a modular system that keeps a real-time feedback with information from different sensors fixed around the 3D printer.

Recently, the great increase of polymers, which are available in FDM technology, such as nylon, TPU (flexible) and soluble plastics makes possible the design of multi-material objects mixing different physical properties (e.g. mechanical, electrical, chemical, optical).
Multi-material 3D printing provides challenges to allow 3D modeling of complex geometric structures and specific appearances. In this context, we are focused on the simulation of bone tissues.

In this paper, we first summarize the state of the art regarding 3D printing utilities and multi-material applications. Then, we describe the features of our control system for remote 3D printing and its hardware requirements. Afterwards we approach the study of multimaterial objects for medical prototypes and finally, we discuss the pros and cons of the results.

The main contributions of this paper are:

- The implantation of a network platform to monitor and modify in real-time the $3 \mathrm{D}$ printing.

- A detailed study about multi-material features towards the $3 \mathrm{D}$ reconstruction of bone tissue.

\section{RELATED WORK}

According to the literature, there are different solutions for remote 3D printing [2]. However, the capabilities of these systems are limited during printing process. The total control for this task is a challenge which has not been resolved until now. The duration of 3D printing jobs depends on the model quality which is closely linked to its layer height. An optimal 3D printing must be supervised. For this reason, recently some webbased systems have been developed to satisfy printing controls. For instance, 3DPrinterOS or Waggle are remote controllers in order to review 3D printers behavior from multiple mobile devices. These under license solutions provide a remote $3 \mathrm{D}$ printing launching 
and a web camera for real-time monitoring. A similar utility is offered by Repetier firmware [3]. It must be installed and configured for a specific 3D printer. Repetier is a standard open-source firmware which includes a web server in order to allow remotely some operation with the $3 \mathrm{D}$ printer through a web interface. However, this solution requires the installation of this specific firmware in the $3 \mathrm{D}$ printer.

In order to expand 3D printing and making it more versatile, we have also studied printing material properties for multi-material solutions [4]. In FDM technology, there are many extruder models that support multimaterial printing such as E3D Kraken or Diamond HotEnd as well as 3D printers which have the capability for mixing different materials like Prusa MK3 or ZMorph among others[5].

In this paper we describe a remote control system for $3 \mathrm{D}$ printing which has been installed in a modular platform. It provides real-time monitoring and the capacity to modify some printer setting during printing process. In addition, we have studied the features of multimaterial printing in order to simulate bone tissues.

\section{REMOTE CONTROL SYSTEM}

In our system we use a combination of open hardware and software, the core of the hardware is a mini computer, specifically a Raspberry $\mathrm{Pi} 3 \mathrm{~B}$, which is a very low cost system focused on teaching in developing areas. Due to its small size and versatility, it has become a key player in many technological projects, both industrial and domestic. Among its main advantages are the great connectivity through several ways(USB, Ethernet, GPIO, WiFi, Bluetooth, etc.), low power consumption, portability, and high performance computing.

In addition, some devices are used to extend the capabilities of the system. Some relays provide us the ability to switch on and off the printers, it is useful as a way to reduce electrical consumption and to launch an unexpected printing job. A web camera per $3 \mathrm{D}$ printer, allow us to see in real time the printing area, in order to check the status of the current model printing. The light sensor installed in the printing room, is capable of turning on a led lamp while the printers are running and there is no enough ambient light. For a better integration of our system, some models have been designed and printed (Figure 1).

In order to speed up remote printing, we have connected a relay to the Raspberry Pi that allows the printer to be switched on and off. This allows you to turn it off, to save energy when you finish a job, or to turn it on without having to go to the office. Several printer control with the same system: Running several Octoprint instances on the same device that listen on different HTTP ports. Each instance connects to the printer with its serial interface over USB. To expose those instances

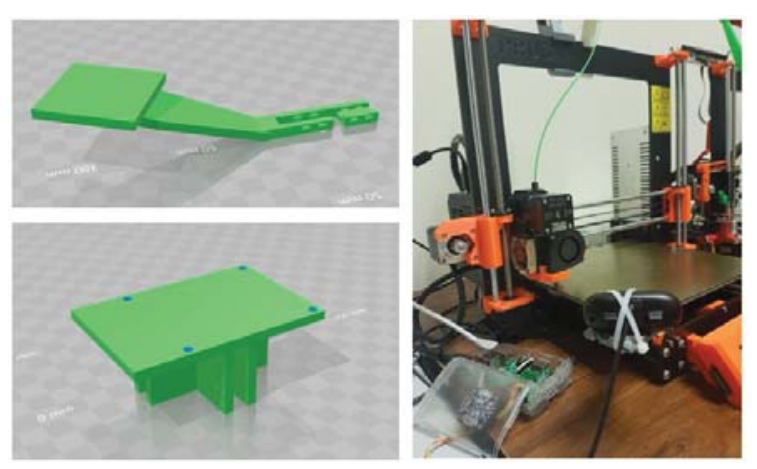

Figure 1: The implantation of our remote control system

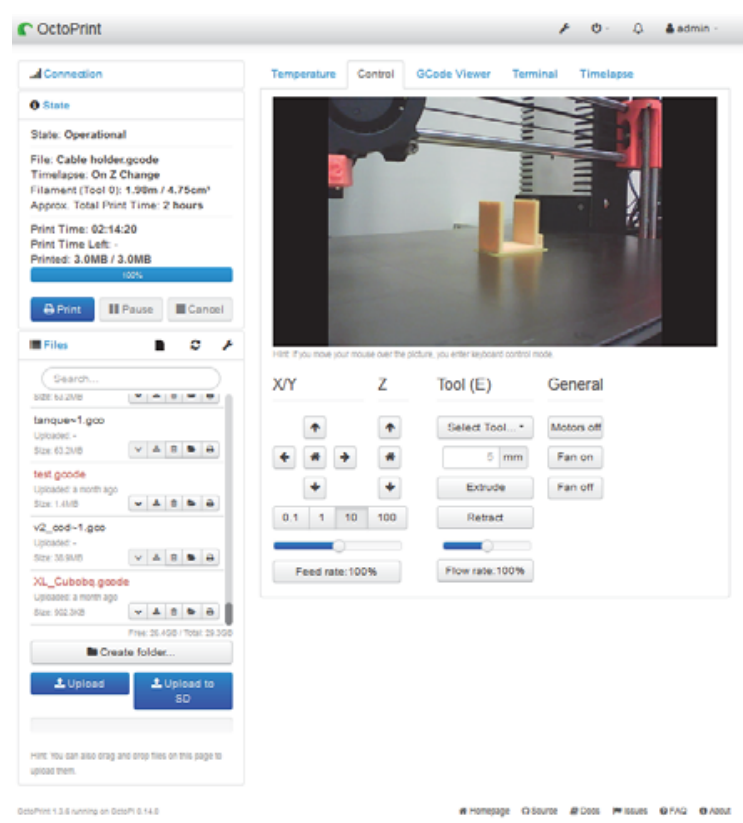

Figure 2: User GUI of Octoprint

to the world using standard HTTPS port, a proxy is used (NGINX). OctoPi is the basis of our system, it is a lightweight Linux distribution, derived from Raspbian. It contains the drivers and dependencies in order to work with almost all commercial 3D printers, and the OctoPrint (Figure 2). Octoprint is a 3D printing server which provides a web interface, allowing 24/7 network access. This software has the the following key features:

- Total control of the printer, axis movement, tools temperature, extruder behavior, etc.

- Real-time view of the printing area.

- Launch, pause, resume and cancel 3D printing.

- Serial communication with the 3D printer, using gcode commands.

- Time-lapse generation that allows to locate and solve printing errors. 


\section{ANALYSIS 3D PRINTING MATERI- ALS}

Since last year, we have been testing a wide variety of 3D materials in order to know their physical properties and their behaviour after printing process. There are several main parameters which must be changed depending the material choice: printing temperature and velocity, infill density, retraction value, etc. [6]. The generation of multi-material models requires a deep analysis about material properties. In this paper, we are focused on the FDM printing materials with a diameter of $1.75 \mathrm{~cm}$.

Many plastics have been tested and today, PLA (polylactic acid) is the best polymer for FDM printing. It has good mechanical properties, easy post-processing and allows faster 3D printings with a high quality. As a negative point, it begins to degrade at a low temperature, $60^{\circ}$. PVA (polyvinyl acetate) is a water soluble substrate for 3D printing that allows for quick, inexpensive, and easy model separation. ABS (acrylonitrile butadiene styrene) is easy to machine and process after printing, with good mechanical properties, but difficult to print. It needs heated bed, emits harmful gases, and is prone to warping. HIPS (High Impact Polystyrene) has similar characteristics to ABS, it can be used as a support in 3D printing, as it dissolves in D-Limonene. But it is very difficult to print in conjunction with $\mathrm{ABS}$, even at similar temperatures, the union layers between them do not adhere sufficiently. FILA-FLEX (mixture of polyurethane and other plastics) can creates parts with a high degree of elasticity and high tensile strength. On the other hand, it has many printing problems, and the printer's extruder may need to be modified. HardenedNYLON: consists of a reinforced nylon, which gives it mechanical, thermal and abrasion resistance, ideal for industrial applications. But it is an abrasive plastic, it should be printed with steel nozzles and not brass.

\section{MULTI-MATERIAL APPLICATION FOR MEDICAL PROTOTYPES}

There are some FDM printers capable to print multimaterial models by many techniques: (1) same materials with different color, (2) different materials, (3) a mix of them. A common practice is printing the support part of models with soluble materials. There are several ways to print multi-material models, the most basic one, is pausing the print in a predefined height and manually changing the plastic filament, but it is impracticable in our project, as it requires the constant attention of an operator.

A multi-extruder configuration, is a common solution to the multi-material printing, it allows to use different materials or colors in the same print. But in our testing, we discover two main disadvantages, multiple plastics can't be mixed at printing time, and the calibration

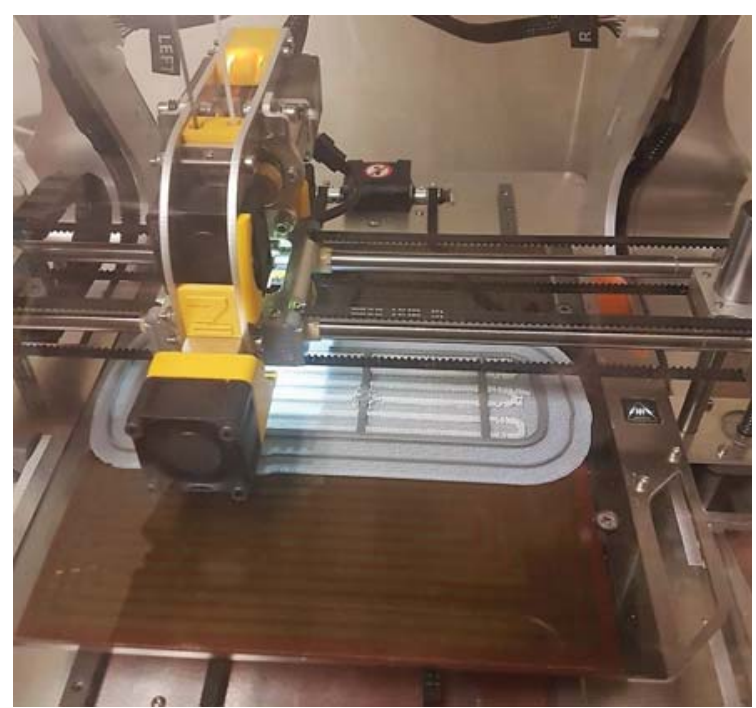

Figure 3: Multi-material prototype with Zmorph printer

phase is challenging, all extruders must be in the same exact height, a difference of a micron results in poorly printed models. The best setup tested is a 3D printer with a single extruder but multiple inputs, it has the benefits of the multi-extruder system, capable of mixing multiple materials, and the calibration is the same no matter how many inputs are present. With a 3 or 4 inputs printer, it is possible to mix different two colours in various proportions to create new colors, combine different plastic, or both. Some test with this configuration, conducted to a model printed with a mixture of ABS and HIPS (Figure 3), results in a printed part that has almost the same strength of the ABS, but it is more elastic.

This set of tests will serve to establish the basis for the creation of models that have the same mechanical characteristics as human bones. In addition, it provides support for a search project focused on the modeling of bone fractures. Multi-material prototypes play a key role in order to simulate physical properties, through different printing materials, of the trabecular and cortial regions of the bones.

\section{CONCLUSIONS AND FUTURE WORK}

3D printing industry has experimented a fast growing around multiple professional areas. Fused Deposition Modelling (FMD) technology allows the use of a wide variety of thermoplastic materials with the possibility of combining between them. In this paper, we have approached two 3D printing challenges: remote control system to monitor and modify in real-time the printing process and the study of material properties in order to generate multi-material solutions. 3D printing can also be used in medical applications such as bone tissue engineering. 
Over our current remote control system and the acquisition of a high knowledge about printing material properties, we are capable to model multi-material bones and explore their mechanical behaviors. This research opens many work lines for 3D printable complex structures with a real-time control during the whole process.

\section{ACKNOWLEDGMENTS}

This work has been partially supported by the Ministerio de Economía y Competitividad and the European Union (via ERDF funds) through the research projects TIN2017-84968-R and DPI2015-65123-R.

\section{REFERENCES}

[1] P. Dudek, "Fdm 3d printing technology in manufacturing composite elements," Archives of Metallurgy and Materials, vol. 58, no. 4, pp. 1415-1418, 2013.

[2] J. Martínez Arrieta, "Improvement and addition of features in $3 \mathrm{~d}$ printing open source software octoprint," 2016.

[3] R. V. Aroca, C. E. Ventura, I. De Mello, and T. F. Pazelli, "Sequential additive manufacturing: automatic manipulation of $3 \mathrm{~d}$ printed parts," Rapid Prototyping Journal, vol. 23, no. 4, pp. 653-659, 2017.

[4] P. Sitthi-Amorn, J. E. Ramos, Y. Wangy, J. Kwan, J. Lan, W. Wang, and W. Matusik, "Multifab: a machine vision assisted platform for multi-material $3 \mathrm{~d}$ printing," ACM Transactions on Graphics (TOG), vol. 34, no. 4, p. 129, 2015.

[5] K. Vidimče, S.-P. Wang, J. Ragan-Kelley, and W. Matusik, "Openfab: a programmable pipeline for multi-material fabrication," ACM Transactions on Graphics (TOG), vol. 32, no. 4, p. 136, 2013.

[6] X. Wang, M. Jiang, Z. Zhou, J. Gou, and D. Hui, "3d printing of polymer matrix composites: A review and prospective," Composites Part B: Engineering, vol. 110, pp. 442-458, 2017. 